\title{
The capability of Several Population-based Approach Software to Analyze Sparse Drug Plasma Concentration Data after Intravenous Bolus Injection
}

\section{Akhmad Kharis Nugroho, ${ }^{{ }^{*}}$ and Lukman Hakim ${ }^{2}$}

1. Department of Pharmaceutics, Faculty of Pharmacy, Universitas Gadjah Mada, Yogyakarta 55281, Indonesia

2. Department of Pharmacology and Clinical Pharmacy, Faculty of Pharmacy, Universitas Gadjah Mada, Yogyakarta 55281, Indonesia

\author{
Info Article \\ Submitted: 14-01-2019 \\ Revised: 10-06-2019 \\ Accepted: 18-06-2019 \\ *Corresponding author \\ Akhmad Kharis Nugroho \\ Email: \\ a.k.nugroho@ugm.ac.id
}

\begin{abstract}
Monolix, NONMEM, and WinBUGS-PKBUGS are among the available software packages for population-based modeling. The sparse-data of drug plasma concentration versus time ( $\mathrm{Cp}$-time) is prevalent in clinically based studies involving patients. It is not ethical in this case, to collect many and large volumes of blood samples. This study was aimed to simulate the capability of Monolix, NONMEM, and WinBUGS-PKBUGS to analyze very sparse $C p$-time data after an intravenous bolus drug administration and to estimate the minimum number of $\mathrm{Cp}$-time data required for an adequate analysis. Data of $C p$-time were obtained based on simulation using the pharmacokinetic one-compartment open model following an intravenous bolus administration of $50 \mathrm{mg}$ of a hypothetical drug. In this respect, six random values of $k$ (rate constant of elimination) and $V d$ (volume of distribution) with mean and standard deviation values of $0.3 \pm 0.1 / \mathrm{h}$ and $30 \pm 10 \mathrm{~L}$, respectively, were used to create simulated $\mathrm{Cp}$-time data of 6 subjects. Simulated $\mathrm{Cp}$-time data in each subject were randomly ranked to choose data based on the intended number of samples in each subject. Several sparse $C p$-time data scenarios, starting from a minimal state, i.e., with a total of $6 \mathrm{Cp}$-time data ( 1 datum per subject) to a rich-data with $48 \mathrm{Cp}$ datapoints ( 8 data per subject), were examined. The goodness-of-fit evaluations, as well as the similarity of individual values of $\mathrm{k}$ and $\mathrm{Vd}$ to the respective real values ( $p>0.05)$, indicate that nonlinear-mixed-effect-model using Monolix, NONMEM, and WinBUGS-PKBUGS can appropriately describe sparse $C p$ time data even with only 2 data per subject. This fact is an important finding to support the demand of analytical tool for a limited number of $\mathrm{Cp}$-time data such as obtained in the therapeutic drug monitoring event.
\end{abstract}

Keywords: Monolix, NONMEM, WinBUGS-PKBUGS, sparse-data, therapeutic drug monitoring

\section{INTRODUCTION}

In 1972, Sheiner and colleague introduced the so-called nonlinear mixed effect model, starting the era of the population-based modeling approach. This approach assumes, a particular parameter or variable, for example, the rate constant of elimination, $\mathrm{K}$, is considered determined by a population or fixed effect value and inter-individual variability, resulting in a different $\mathrm{K}$ parameter value in each subject. This approach directly focuses on the population data, allowing analyses of the sparse-data commonly faced in clinical studies involving patients (Sheiner et al., 1972). This approach also allows the correlation of a particular parameter such as clearance (CL), distribution volume (Vd) or elimination rate constant $(\mathrm{K})$ to specific covariates such as sex, age, body weight, or serum creatinine in a quantitative manner (Jonsson and Karlsson, 1998; Wählby et al., 2001). NONMEM is the first (Sheiner et al., 1972) and the gold standard (Frame, 2006; Keizer et al., 2013) software in populationbased approach, allowing quantitative analyses of the clinical data such as in a therapeutic drug 
monitoring (TDM) (Shaker et al., 2013). NONMEM provides fast, robust, accurate, and precise computation (Plan et al., 2012). The first-order conditional estimation (FOCE) with interaction is a commonly used estimation method in NONMEM (Wang, 2007).

Several free or free-for-academic alternative tools for population modeling are available. Monolix is an example of the free-for-academic software, developed since 2003. The computation is based on SAEM (Stochastic Approximation Effect Model) algorithm using MATLAB library engine (Chan et al., 2011) developed based on a thorough statistical theory, providing a fast and efficient calculation (Lavielle and Mentré, 2007). Monolix, which is currently maintained by Lixoft company, provides an excellent graphical user interface, facilitating a more practical application (Lavielle, 2014).

Another free tool in population pharmacokinetic-pharmacodynamic analyses is PKBUGS running under WinBUGS environment (Lunn et al., 2002). WinBUGS is a general Bayesian modeling framework that can be used to analyze several different purposes and applications, using Markov chain Monte Carlo (MCMC) techniques. Bayesian inference Using Gibbs Sampling (BUGS) project has been initiated by MRC Biostatistics Unit, at University of Cambridge, in collaboration with Imperial College School of Medicine at St Mary's, London (Lunn et al., 2002; Ntzoufras, 2011).

Recently, we have reported the capability of a population-based approach to analyze limited data in the per-oral administration scenario (Nugroho et al., 2017). However, it is not yet clear to what extent of sparse-condition can be adequately described, mainly using several different administration routes. This research was aimed to analyze very sparse Cp-time data after an intravenous bolus drug administration and to estimate the minimum number of $\mathrm{Cp}$-time data required for an adequate analysis.

\section{MATERIAL AND METHODS}

\section{Preparation of Data simulation}

We simulated the data of $\mathrm{Cp}$ versus time after intravenous-bolus administration of $50 \mathrm{mg}$ of a hypothetical compound using Microsoft ${ }^{\circledR}$ Excel $^{\circledR}$ 365 (running on Windows 10 machine) (Liengme, 2015) based on the one-compartment open model with intravenous bolus injection for Cp (Welling, 1986) as presented in Equation 1.

$C p=\frac{\text { Dose }}{V d} \cdot e^{-K t}$
This step resulted in the creation of the richdata of $\mathrm{Cp}$, i.e., 48 data point of 6 subjects at eight time points post dosing (at $0.25 ; 0.5 ; 1 ; 2 ; 3 ; 4 ; 6$ and 8h). Each individual-parameter of $\mathrm{K}$ and $\mathrm{Vd}$ was randomly selected based on the mean and standard of deviation of $0.3 \pm 0.1 / \mathrm{h}$ and $30 \pm 10 \mathrm{~L}$ (Figure $1 \mathrm{~A}$ ). Furthermore, the sparse-data were originated based on a random selection of the rich-data by choosing 2 and 1 data/datum per subject. This step resulted in 12 data points and 6 data points from 6 subjects (Figure $1 \mathrm{~B}$ and $\mathrm{C}$ ).

\section{Data analysis}

Monolix (stand alone, version 2018R2, running on Windows 10 machine), WinBUGS (version 1.3 and 1.4) - PKBUGS (version 1.0), and NONMEM (version 7.4, using Gfortran compiler, directed using PLTTools lite version 6) (Frame, 2006) were used to model the rich-data and the sparse-data. The structural model to describe the simulated $\mathrm{Cp}$ data by intravenous bolus administration was based on the onecompartmental open model, as presented in Equation 1. Moreover, the inter-individual variability is determined by an exponential error model (equation 2).

$P_{i}=\theta \cdot \exp \left(\eta_{i}\right)$

Term $\theta$ is the population value, or the fixed effect parameter of $P . P_{i}$ is the individual estimate value, and $\eta_{\mathrm{i}}$ is the inter-individual variation, assumed to be independently and normally distributed with mean zero and variance $\omega^{2}$. The interindividual variability was applied for $\mathrm{K}$ and $\mathrm{Vd}$. The residual error is described by the additive error model (equation 3).

$F p_{i j}=F o_{i j}+\varepsilon_{1}$

Term $\mathrm{Fp}_{\mathrm{ij}}$ is the prediction of the $\mathrm{j}^{\text {th }}$ evaluated functions ( $\mathrm{Cp}$ ). $\mathrm{Fo}_{\mathrm{ij}}$ is the measured value of the evaluated function (Cp), and $\varepsilon$ represents the residual deviation of the predicted from the observed value and is assumed to be independent and distributed with mean zero and variance $\sigma^{2}$. The analysis of the population parameters $\theta, \omega^{2}$, and $\sigma^{2}$ was performed using the SAEM algorithm (Monolix), MCMC (WinBUGS-PKBUGS), and the first-order conditional estimation (FOCE) with interaction method (NONMEM).

In Monolix, analyses were performed using the structural one compartment intravenous bolus model with $\mathrm{K}$ and Vd parameters provided in Monolix library. No covariate was applied while the covariance implemented the default diagonal pattern. 
Table I. The comparison of the individual parameter of $\mathrm{K}$ and Vd of the rich-data, the sparse-data with 12 data-points (2 data per subject) and the sparse-data with 6 data-points (one datum per subject) estimated by Monolix, NONMEM, and WinBUGS-PKBUGS (data are presented as mean \pm standard of deviation).

\begin{tabular}{ccccccc}
\hline \multirow{2}{*}{ Data } & \multicolumn{3}{c}{ K (per hour) } & \multicolumn{3}{c}{ Vd (L) } \\
\cline { 2 - 7 } & Monolix & NONMEM & WinBUGS-PKBUGS & Monolix & NONMEM & WinBUGS-PKBUGS \\
\hline rich-data & $0.3 \pm 0.1$ & $0.3 \pm 0.1$ & $0.3 \pm 0.1$ & $30.0 \pm 11.0$ & $30.0 \pm 11.0$ & $30.0 \pm 11.0$ \\
* sparse-12 $^{*} 0.3 \pm 0.1$ & $0.3 \pm 0.1$ & $0.3 \pm 0.0$ & $29.2 \pm 9.4$ & $29.1 \pm 9.4$ & $28.9 \pm 8.9$ \\
** sparse- $6^{0}$ & $0.5 \pm 0.1^{\#}$ & $0.4 \pm 0.0^{\#}$ & $0.4 \pm 0.1^{\#}$ & $14.9 \pm 0.3^{\#}$ & $18.4 \pm 7.0^{\#}$ & $16.7 \pm 1.4^{\#}$ \\
\hline
\end{tabular}

* Sparse-data with 12 data-points ( 2 data per subject); ** Sparse-data with 6 data-points (1 datum per subject); \# Mean parameter values were significantly different from the respective reference ones $(\mathrm{p}<0.05)$

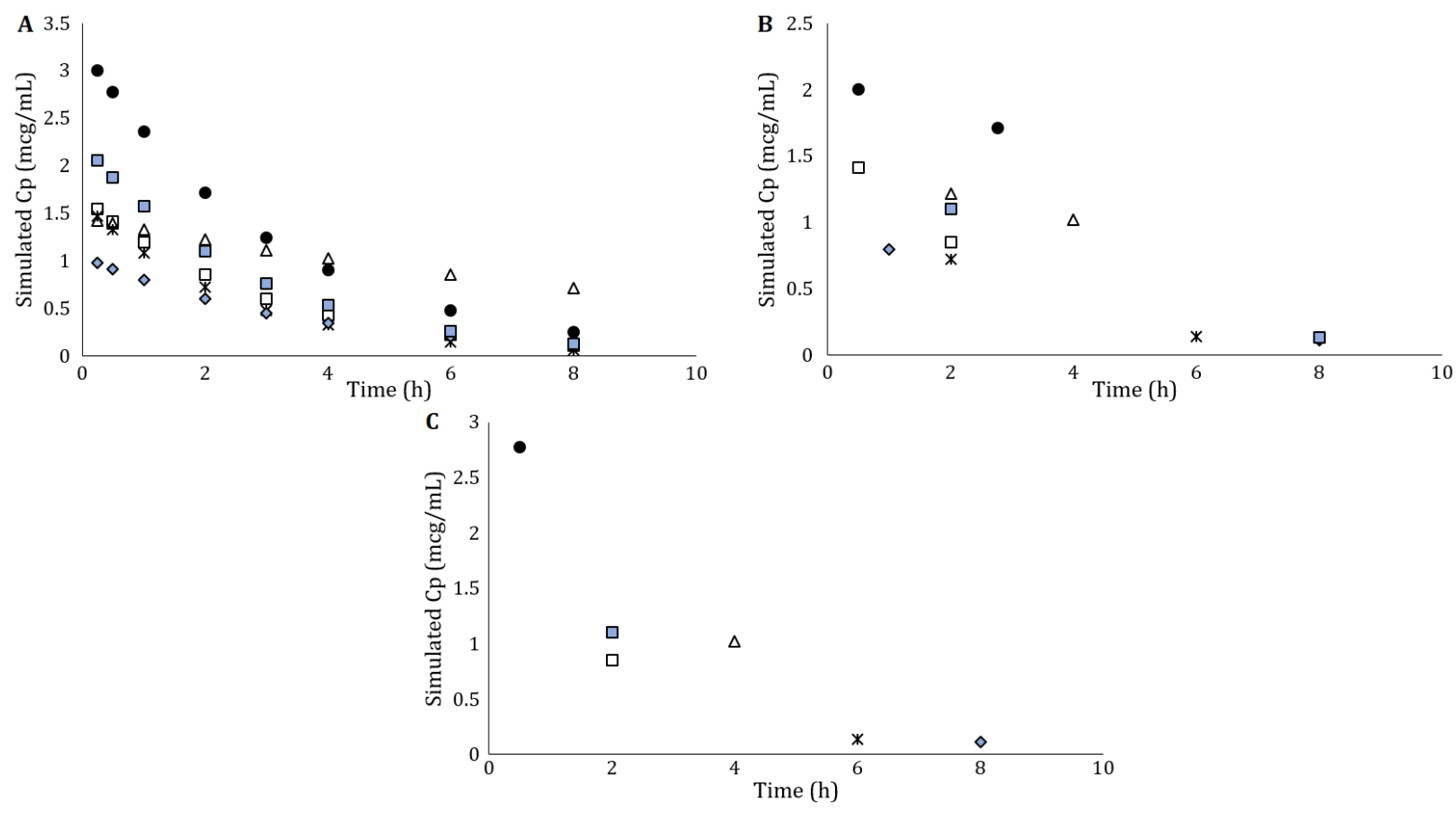

Figure 1. Data of the rich-data (panel A) and the sparse-data with 12 data-points ( 2 data per subject) (panel B) and the sparse-data with 6 data-points (1 datum per subject) (panel C) of plasma concentration (Cp) versus time profile of a $50 \mathrm{mg}$ theoretical compound delivered by an intravenous bolus injection, simulated in 6 subjects (1: filled-circle, 2: open-triangle, 3: star, 4: open-square, 5: filled-diamond, 6: filled-square).

Data were assumed to follow a log-normal distribution. The calculation was performed using $0.3 / \mathrm{h}$ and $30 \mathrm{~L}$ as initial estimates of $\mathrm{K}$ and $\mathrm{Vd}$, respectively. The initial estimates of the random error (eta) were 0.1 , and 0.1 for $\mathrm{K}$ and $\mathrm{Vd}$, respectively. Additive error model was calculated, with the initial values of sigma of 0.001 .

Modeling in NONMEM was performed using the provided one-compartment oral model (ADVAN 1 trans 1) with $\mathrm{K}$ and $\mathrm{Vd}$ parameters provided in NONMEM PK library. No covariate was applied while the covariance implemented the default diagonal pattern. The calculation was performed using $0.3 / \mathrm{h}$ and $30 \mathrm{~L}$ as initial estimates of $\mathrm{K}$ and $\mathrm{Vd}$, respectively. The initial estimates of the random error (eta) were 0.1 and 0.1 for both $\mathrm{K}$ and Vd. Additional residual error model was used, with the initial values of 0.1 .

Analyses in WinBUGS were performed using the structural one compartment intravenous bolus model with clearance $(\mathrm{Cl})$ and $\mathrm{Vd}$ parameters provided in PKBUGS add-in. No covariate was applied. The calculation was performed using $15 \mathrm{~L} / \mathrm{h}$ and $30 \mathrm{~L}$ as initial estimates of $\mathrm{Cl}$ and $\mathrm{Vd}$, respectively. The initial estimates of the random error (eta) were $10 \%$ for both $\mathrm{Cl}$ and $\mathrm{Vd}$. 

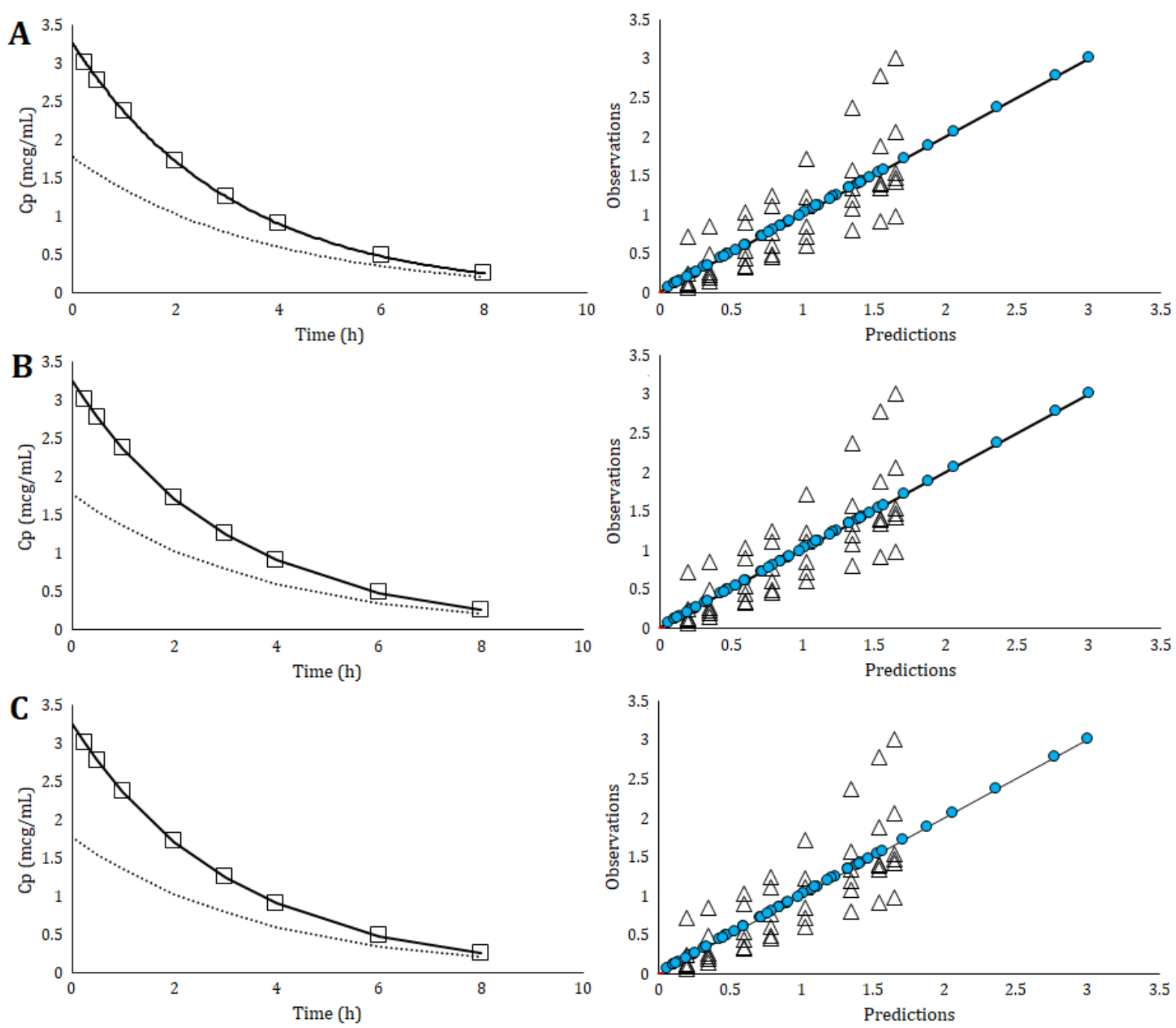

Figure 2. The goodness-of-fit evaluation of the rich-data of $\mathrm{Cp}$ in Monolix (A), NONMEM (B) and WINBUGSPKBUGS (C) i.e., 1) the typical examples of individual fitting (subject number 1) of the "observed" Cp data (DV) with the individual (solid-curve) and population model (dotted-curve) predictions (left panels), and 2) the correlation of DV versus population model prediction (open-triangle) and the individual model prediction of $\mathrm{Cp}$ (closed-circle) (right panels).

Parameter $\mathrm{K}$ was estimated based on the estimation of $\mathrm{Cl}$ and $\mathrm{Vd}$ values. While PKBUGS is running in WinBUGS 1.3 , to obtain more flexible MCMC, the final model was exported to WinBUGS 1.4 platform using "Print model" menu.

The adequacy of modeling in all cases was analyzed based on the goodness-of-fit evaluations. These evaluations consisted of 1) the individual fitting with the individual and population model prediction curves; 2 ) the correlation of DV, namely the dependent variable (the "observed" Cp) versus population model prediction of $\mathrm{Cp}$ and the individual model prediction of $\mathrm{Cp}$. Such evaluations are considered crucial to judge the adequacy of specific modeling analyses (Mohammed et al., 2012; Owen and Fiedler-Kelly, 2014; Zheng et al., 2014).

The post-hoc individual parameter values of $\mathrm{K}$ and Vd obtained from the analyses of the sparsedata using Monolix, WinBUGS-PKBUGS, and NONMEM were compared to the respective individual values of the parameters. Due to a normal distribution of all data tested based on Shapiro-Wilkes method using OpenStat 2014 

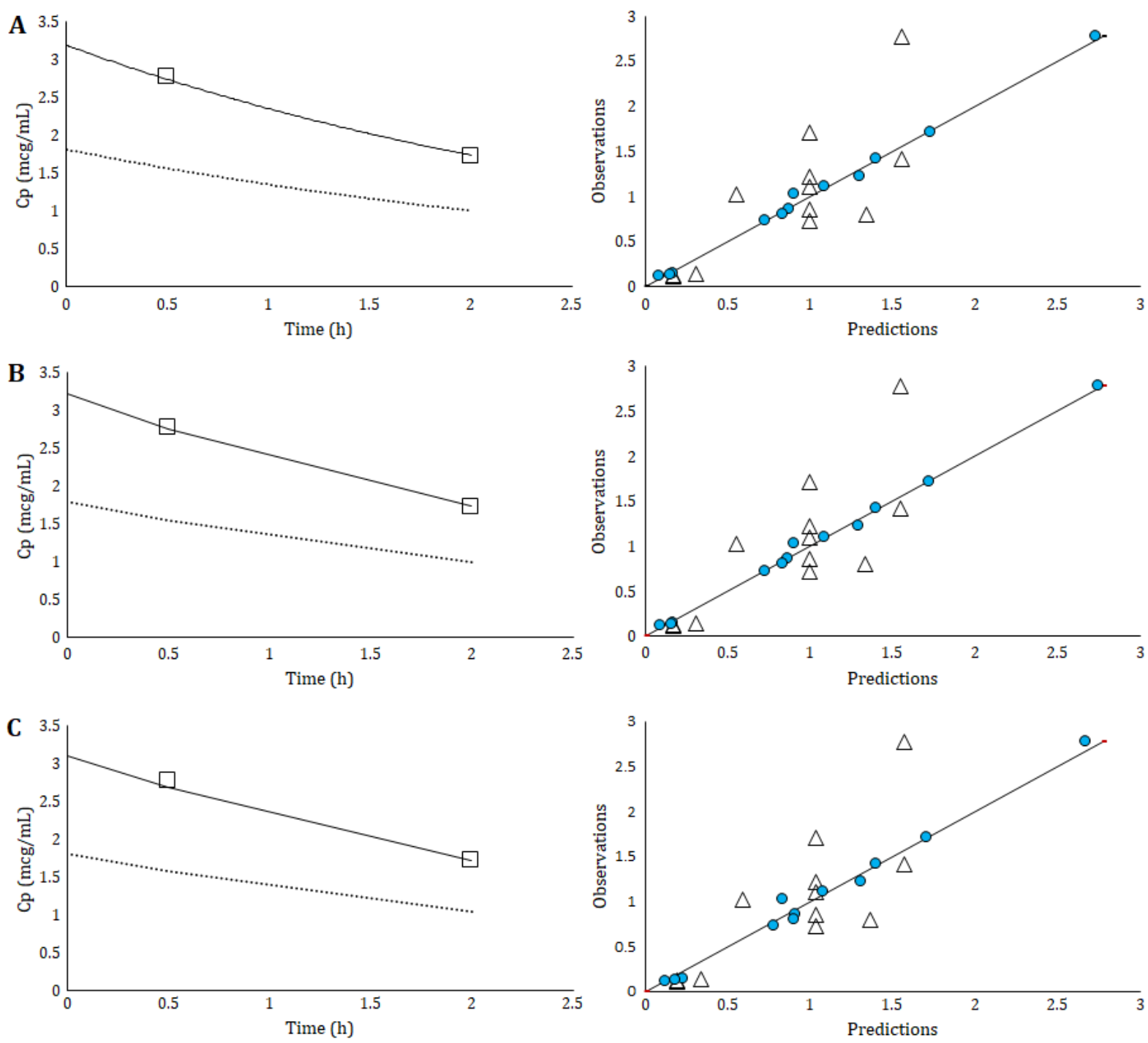

Figure 3. The goodness-of-fit evaluation of the sparse-data with 2 data per subject of $\mathrm{Cp}$ in Monolix (A), NONMEM (B) and WINBUGS-PKBUGS (C) i.e. 1) the typical examples of individual fitting (subject number 1) of the "observed" Cp data (DV) with the individual (solid -curve) and population model (dotted-curve) predictions (left panels), and 2) The correlation of DV versus population model prediction (open-triangle) and the individual model prediction of $\mathrm{Cp}$ (closed-circle) (right panels).

(Miller, 2012), the comparison of the mean values of $\mathrm{K}$ and $\mathrm{Vd}$ of the sparse-data to the rich-data values were performed based on the paired t-test method using Microsoft ${ }^{\circledR}$ Excel $^{\circledR}$ 365.

\section{RESULTS AND DISCUSSION}

Although each population-based modeling software package has a different of complex calculation algorithms, the goodness-of-fit evaluations demonstrate that all the packages, i.e., Monolix,NONMEM,andWinBUGS-PKBUGS properly analyze the rich-data as well as the sparse-data with 12 or even 6 data-points. In all cases, a proper population fitting of the $\mathrm{Cp}$ data is indicated by the absence of a specific pattern or shape such as sigmoid, or shoe shapes. Moreover, the individual prediction indicates an ideal situation, where most of the DV and individual-prediction of $\mathrm{Cp}$ coincide on the line of identity. The analyses in Monolix, NONMEM and WinBUGS-PKBUGS, are presented in Figure 2 (the rich-data of $\mathrm{Cp}$ ), Figure 3 (the sparse - 12 data points), and Figure 4 (the sparse 6 data points). 

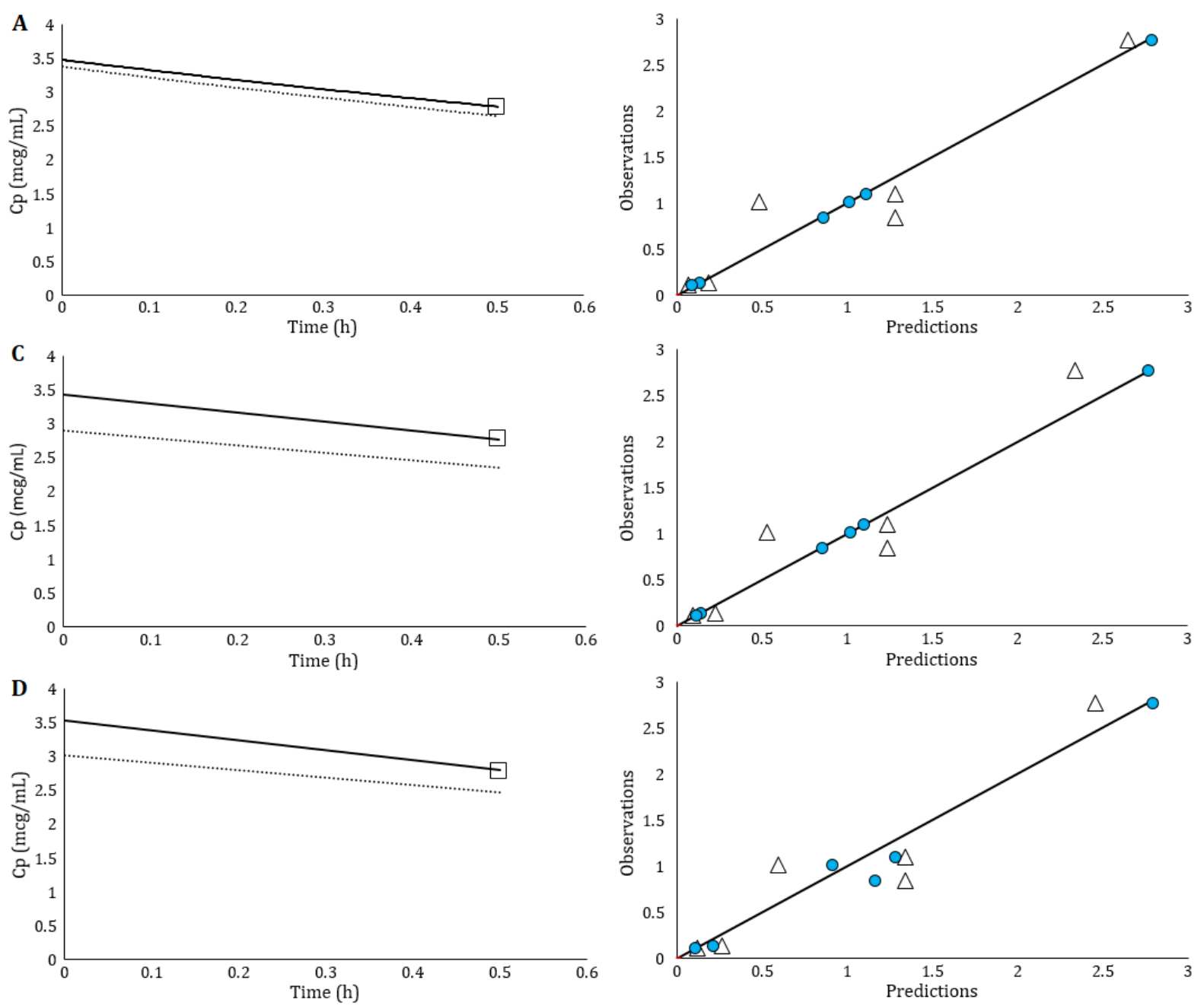

Figure 4. The goodness-of-fit evaluation of the sparse-data with 1 datum per subject of Cp in Monolix (A), NONMEM (B) and WINBUGS-PKBUGS (C) i.e. 1) the typical examples of individual fitting (subject number 1) of the "observed" Cp data (DV) with the individual (solid-curve) and population model (dotted-curve) predictions (left panels), and 2) the correlation of DV versus population model prediction (open-triangle) and the individual model prediction of $\mathrm{Cp}$ (closed-circle) (right panels).

Furthermore, we analyzed the individual pharmacokinetic parameter values of $\mathrm{K}$ and $\mathrm{Vd}$ of the sparse-data to the respective reference $\mathrm{K}$ and Vd values used to simulate the Cp data, based on a paired t-test (Table I). In all comparisons, the $\mathrm{K}$ and Vd values estimated by using Monolix, NONMEM and WinBUGS-PKBUGS of the sparse-data with 12 data-points are similar to the respective reference values $(\mathrm{p}>0.05)$. Moreover, although the fitting analyses are adequate with all software packages, the estimated $\mathrm{K}$ and $\mathrm{Vd}$ values of the sparse-data with 6 data-points are significantly different from the reference values $(\mathrm{p}<0.05)$. The average percentage ratios of $K$ values of the sparse 6 data- points to the reference values are in the range of 188\% (Monolix), 184\% (NONMEM) and 161\% (WinBUGS-PKBUGS). Similarly, such percentage ratios of $\mathrm{Vd}$ values are in the range of $56 \%$ (Monolix), 69\% (NONMEM), and 62\% (WinBUGSPKBUGS).

Those facts demonstrate that sparse-data obtained with a limited number of subjects (6) and a limited number of samples (2) per subject, can be appropriately fitted in all cases. In agreement with our previous report with the sparse per-oral $\mathrm{Cp}$ data (Nugroho et al., 2017), the conditions again highlight the power of population modeling to analyze the sparse-data. This finding is important, 
concerning the possibility of an accurate analysis with minimal data obtained in clinical studies involving patients such as in the case of a therapeutic drug monitoring of certain drugs. In such instances, sparse-data are collected from patients (Parke and Charles, 1998; Shaker et al., 2013). The conventional approach with a two-stage approach cannot analyze such conditions as this classical method requires a rich-data situation (Nugroho et al., 2017).

We could consider that, with the case of an intravenous bolus injection of a compound following a one-compartmental open model, the minimum data-points of population modeling analyses is 12 . However, we should realize that it could be related to the less complexity of the model, with only 2 model parameters ( $\mathrm{K}$ and $\mathrm{Vd}$ ), and without the involvement of any covariate for the analyses. In modeling cases involving more parameters, with or without any covariates, the calculation is more complicated (Mould and Upton, 2013), and the minimum number of samples might be different. It is important to find such guidance in estimating the minimum samples required for those cases, including per-oral administration of a compound following a multi-compartment open model. This information will be helpful in the preparation of an in vivo studies in patients. The appropriate scenarios can be proposed to ascertain a valid analysis of the limited data

Furthermore, the fact that Monolix, NONMEM, and WinBUGS-PKBUGS demonstrated similar power in population modeling is also essential, especially for the future of the extensive application of population-modeling in Indonesia and other developing countries. It is related to the limited budget allocation support in most institutions to buy an annual and relatively expensive license cost of the commercial software package. Monolix is free for any educational use, while WinBUGS-PKBUGS is an entirely freesoftware package for everyone willing to perform a population-based approach based on Bayesian Statistics implementing an MCMC method (Lunn et al., 2002).

\section{CONCLUSION}

Population-based approach appropriately describes the sparse-data in an intravenous bolus injection scenarios. Such capability is essential to achieve the ideal therapeutic outcome based on therapeutic drug monitoring. Monolix (free-foracademic) and WinBUGS-PKBUGS (completely free) software packages have comparable performances to NONMEM, the gold standard of population based software. The possibility to use a completely free software package can facilitate a more extensive application of population modeling to facilitate an optimum therapeutic outcome in Indonesia.

\section{ACKNOWLEDGMENT}

This research was supported by Hibah Penelitian Dasar Unggulan Perguruan Tinggi, a research grant scheme provided by the Ministry of Research, Technology and Higher Education, the Republic of Indonesia, the year of 2018.

\section{REFERENCE}

Chan PLS., Jacqmin P., Lavielle M., McFadyen L. and Weatherley B., 2011. The use of the SAEM algorithm in MONOLIX software for estimation of population pharmacokineticpharmacodynamic-viral dynamics parameters of maraviroc in asymptomatic HIV subjects. J. Pharmacokinet. Pharmacodyn. 38, 41-61. https://doi.org/10.1007/s10928-0109175-z

Frame B., 2006. Mixture Modeling with NONMEM V, in: Ette, E.I., Williams, P.J. (Eds.), Pharmacometrics: The Science of Quantitative Pharmacology. John Wiley \& Sons, Inc, Hoboken, New Jersey, pp. 723-757.

Jonsson EN. and Karlsson MO., 1998. Automated covariate model building within NONMEM. Pharm. Res. 15, 1463-1468.

Keizer RJ., Karlsson MO. and Hooker A., 2013. Modeling and Simulation Workbench for NONMEM: Tutorial on Pirana, PsN, and Xpose. CPT Pharmacomet. Syst. Pharmacol. 2, e50. https://doi.org/10.1038/psp.2013.24

Lavielle M., 2014. Mixed Effects Models for the Population Approach: Models, Tasks, Methods and Tools. CRC Press.

Lavielle M. and Mentré F., 2007. Estimation of Population Pharmacokinetic Parameters of Saquinavir in HIV Patients with the MONOLIX Software. J. Pharmacokinet. Pharmacodyn. 34, 229-249. https://doi.org/10.1007/s10928-0069043-z

Liengme B., 2015. A Guide to Microsoft Excel 2013 for Scientists and Engineers. Academic Press.

Lunn DJ., Best N., Thomas A., Wakefield J. and Spiegelhalter D., 2002. Bayesian analysis of population PK/PD models: general concepts 
and software. J. Pharmacokinet. Pharmacodyn. 29, 271-307.

Miller W., 2012. OpenStat Reference Manual. Springer Science \& Business Media.

Mohammed BS., Engelhardt T., Cameron GA., Cameron L., Hawksworth GM., et al., 2012. Population pharmacokinetics of single-dose intravenous paracetamol in children. $\mathrm{Br}$. J. Anaesth. 108, 823-829. https://doi.org/10.1093/bja/aes025

Mould DR. and Upton RN., 2013. Basic Concepts in Population Modeling, Simulation, and Model-Based Drug Development-Part 2: Introduction to Pharmacokinetic Modeling Methods. CPT Pharmacomet. Syst. Pharmacol. 2, e38. https://doi.org/10.1038/psp.2013.14

Ntzoufras I., 2011. Bayesian Modeling Using WinBUGS. John Wiley \& Sons.

Nugroho AK., Hakim AR. And Hakim L., 2017. Population-Based Approach to Analyze Sparse Sampling Data in Biopharmaceutics and Pharmacokinetics using Monolix and NONMEM. Indones. J. Pharm. 28, 205. https://doi.org/10.14499/indonesianjphar m28iss4pp205

Owen JS. and Fiedler-Kelly J., 2014. Introduction to population pharmacokinetic/pharmacodynamic analysis with nonlinear mixed effects models. Wiley, Hoboken, New Jersey.

Parke J. and Charles BG., 1998. NONMEM Population Pharmacokinetic Modeling of Orally Administered Cyclosporine From Routine Drug Monitoring Data After Heart Transplantation: Ther. Drug Monit. 20, 284293. https://doi.org/10.1097/00007691199806000-00008
Plan EL., Maloney A., Mentré F., Karlsson MO. And Bertrand J., 2012. Performance comparison of various maximum likelihood nonlinear mixed-effects estimation methods for doseresponse models. AAPS J. 14, 420-432. https://doi.org/10.1208/s12248-0129349-2

Shaker E., Hamadi S., Idkaidek N., Blakey EG. and AlSaleh A., 2013. Therapeutic Drug Monitoring and Population Pharmacokinetics of Digoxin in Jordanian Patients. Am. J. Pharmacol. Sci. 1, 15-21. https://doi.org/10.12691/ajps-12-1

Sheiner LB., Rosenberg B. and Melmon KL., 1972. Modelling of individual pharmacokinetics for computer-aided drug dosage. Comput. Biomed. Res. 5, 411-459.

Wählby U., Jonsson EN. and Karlsson MO., 2001. Assessment of actual significance levels for covariate effects in NONMEM. J. Pharmacokinet. Pharmacodyn. 28, 231-252.

Wang Y., 2007. Derivation of various NONMEM estimation methods. J. Pharmacokinet. Pharmacodyn. 34, 575-593. https://doi.org/10.1007/s10928-0079060-6

Welling PG., 1986. Graphic methods in pharmacokinetics: the basics. J. Clin. Pharmacol. 26, 510-514. https://doi.org/10.1002/j.15524604.1986.tb02943.x

Zheng S., Matzneller P., Zeitlinger M. and Schmidt S., 2014. Development of a Population Pharmacokinetic Model Characterizing the Tissue Distribution of Azithromycin in Healthy Subjects. Antimicrob. Agents Chemother. 58, 6675-6684. https://doi.org/10.1128/AAC.02904-14 